\title{
SOBRE CÓMO UN EDIFICIO VULNERABLE RESISTIÓ SIN DAÑOS EL SISMO DE CARACAS DE 1967
}

\author{
Alejandra Guerrero ${ }^{(1)}$ y Oscar A. López ${ }^{(2)}$
}

\begin{abstract}
RESUMEN
El sismo de 1967 ocasionó daños en el 48\% de los edificios localizados en la zona de Los Palos Grandes-Altamira en Caracas. Se analiza el sorprendente desempeño de un edificio de marcos de concreto armado, de elevada vulnerabilidad, el cual no sufrió daños durante el sismo a pesar de no poseer vigas en una dirección y de tener una condición de piso débil en su nivel inferior debido a la ausencia de paredes. Se generaron cuatro pares de acelerogramas representativos del movimiento en la zona de gran espesor de sedimentos considerando que el movimiento tiene una intensidad en la dirección Norte-Sur aproximadamente tres veces mayor que en la dirección Este-Oeste. Se desarrollaron modelos matemáticos ajustados a las mediciones de vibración ambiental. La respuesta del edificio fue determinada mediante análisis dinámico no lineal, obteniéndose un desempeño similar al observado, resaltando una variación significativa en la respuesta ante un acelerograma u otro. Destaca la influencia determinante que tuvo la orientación del edificio; la carencia de daños se debió a la coincidencia favorable entre la dirección de mayor rigidez y resistencia de la estructura y la dirección de mayor intensidad del movimiento. Se evaluó el desempeño del edificio si se hubiese construido rotado $90^{\circ}$, obteniéndose daño estructural importante, lo cual enfatiza la necesidad de reforzar edificios antiguos similares que no sufrieron daños.
\end{abstract}

Palabras clave: sismo 1967; edificio Belaire; análisis dinámico no lineal; Los Palos Grandes.

\section{ABOUT HOW A VULNERABLE BUILDING RESISTED THE 1967 CARACAS EARTHQUAKE WITHOUT DAMAGE}

\begin{abstract}
The 1967 earthquake caused damage in $48 \%$ of buildings located in the Los Palos Grandes-Altamira area in Caracas. The surprising performance of a highly vulnerable reinforced concrete frame building is analyzed, which did not suffer damage during the earthquake despite not having beams in one direction and having a weak floor condition at its lower level due to the absence of walls. Four pairs of accelerograms representative of movement in the thick sediment zone were generated, considering that the motion has an intensity in the North-South direction that is approximately three times greater than the East-West direction. Mathematical models adjusted to environmental vibration measurements were developed. The response of the building was determined by means of non-linear dynamic analysis, achieving a performance similar to that observed, highlighting a significant
\end{abstract}

\footnotetext{
Artículo recibido el 27 de enero de 2021 y aprobado para su publicación el 12 de octubre de 2021. Se aceptarán comentarios y/o discusiones hasta cinco meses después de su publicación.

(1) Facultad de Ingeniería, Universidad Central de Venezuela, Ciudad Universitaria de Caracas, Los Chaguaramos, Caracas, Venezuela, C.P. 1053. guerrerocale@gmail.com

(2) IMME, Facultad de Ingeniería, Universidad Central de Venezuela, Los Chaguaramos, Caracas, Venezuela; oalsf@yahoo.com
} 
variation in the response from one accelerogram to another. The influence of the particular orientation of this building is highlighted; the lack of damage was due to the favorable coincidence between the strong direction of greater rigidity and resistance of the structure and the direction of greater intensity of ground motion. The performance of the building was evaluated if it had been built rotated $90^{\circ}$, obtaining significant structural damage, which emphasizes the need to reinforce similar old buildings that were not damaged.

Keywords: 1967 earthquake; Belaire building; nonlinear dynamic analysis; Los Palos Grandes.

\section{INTRODUCCIÓN}

El terremoto de Caracas de 1967 que ocasionó daños estructurales en el 20\% de los edificios en Los Palos Grandes-Altamira (LPG-A) y algún tipo de daño en casi la mitad de los edificios de la zona, ha sido estudiado por diversos autores, CPES, (1978), Grases (1968), Sozen et al. (1968), Skinner (1969) y otros, pero más recientemente por Urich (2013). Cuatro edificios (10-12 pisos) se derrumbaron y muchos otros se dañaron, debido principalmente a la amplificación del movimiento en los sedimentos profundos y a la elevada vulnerabilidad de sus estructuras diseñadas en los años 1950-1967 con normas sísmicas insuficientes. La vulnerabilidad era aún mayor en muchos de estos edificios debido a la práctica constructiva de la época de no colocar vigas altas, o colocarlas en una sola dirección, dejando la dirección ortogonal con vigas planas o sin vigas, con solo la losa contribuyendo a la resistencia y rigidez lateral y con la dimensión débil de las columnas orientada en esa misma dirección. Adicionalmente, a ello se sumaba la presencia de paredes de bloques de arcilla en los pisos superiores que se descontinuaban en la planta baja generando un entrepiso blando y débil. En la actualidad existe un número importante de edificios similares en la ciudad, por lo que conviene conocer las razones de su desempeño en 1967, sea este adecuado o inadecuado, bajo el marco de la nueva información desarrollada en los 54 años transcurridos desde 1967.

Varios otros edificios localizados en la misma zona de LPG-A no sufrieron daños en 1967, a pesar de tener alturas y estructuras similares y ser diseñadas con las mismas normas, como es el caso del edificio Belaire. Urich y López (2006) señalan que fue la ausencia de planos resistentes bien definidos en una dirección, una de las razones del mal desempeño de edificios altos. Dichos autores concluyen que la aceleración máxima del terreno no ha debido superar valores entre $0,02 \mathrm{~g}$ y $0,03 \mathrm{~g}$ en dirección Este-Oeste (E-O) para que no se hayan presentado daños en este edificio.

El objetivo de esta investigación es determinar las razones por las cuales el edificio Belaire, con características similares a los derrumbados, no sufrió daños. A diferencia del estudio previo de Urich y López (2006) que usaron el espectro normativo nacional y análisis lineal elástico, en esta investigación se utilizan acelerogramas representativos del sismo de 1967, desarrollados previamente por los autores con información sismológica y local actualizada (Guerrero y López, 2019), se calibran los modelos matemáticos con ensayos de vibración ambiental y se aplican métodos de análisis no lineal. Esta investigación constituyó parte del trabajo de grado de M.Sc. del primer autor bajo la tutoría del segundo autor (Guerrero, 2018).

\section{MOVIMIENTO SÍSMICO EN LOS PALOS GRANDES EN 1967}

Al sismo de 1967 se le asignó una magnitud $\mathrm{M}_{\mathrm{w}}=6,6 \mathrm{y}$ una profundidad focal de $14 \mathrm{~km}$, estimando el plano de falla a aproximadamente $29 \mathrm{~km}$ de LPG-A (Guerrero y López, 2019). No hubo registros acelerográficos del evento. La zona LPG-A se caracteriza por la presencia de sedimentos densos de hasta $360 \mathrm{~m}$ de profundidad con un valor de VS30 de $300 \mathrm{~m} / \mathrm{s}$, definido como suelo intermedio en la 
microzonificación de la ciudad (Schmitz, et al, 2011). Para los propósitos de este artículo, este sitio se denomina como suelo profundo. En un estudio previo (Guerrero y López, 2019), usando modelos de atenuación que incorporan efectos de cuenca (Bozorgnia et al, 2014), los autores obtuvieron espectros de aceleración para el movimiento más probable ocurrido en el sitio. La Figura 1 muestra los espectros resultantes, en suelo profundo y en roca, para las direcciones N-S y E-O. El movimiento en dirección N-S se estima 3 veces más intenso que en la dirección E-O, como lo señala el registro del sismoscopio mostrado en la Figura 1a; éste fue el único registro del evento sísmico, obtenido en roca en Caracas, el cual señala la mayor intensidad en dirección N-S, lo cual fue también destacado por otros autores con base a la observación del desempeño de edificios (Sozen et al., 1968).

La aceleración obtenida es $0,12 \mathrm{~g}$ para la dirección $\mathrm{N}-\mathrm{S}$ en roca, pero aumenta hasta $0,19 \mathrm{~g}$ en la superficie del depósito. Comparando la Figura 1.c (roca) con 1.b (suelo profundo) se aprecia el efecto de amplificación del movimiento debida a la profundidad y rigidez de los sedimentos. El cociente entre la aceleración espectral en suelo profundo (Figura 1.b) y la aceleración en roca (Figura 1.c) varía con el período de vibración, pero es del orden de 3,5 en el rango entre 1 s y 2 s, destacando así la mayor amplificación en la banda de períodos asociados a edificios de mediana altura (Guerrero y López, 2019).

a) Sismoscopio.

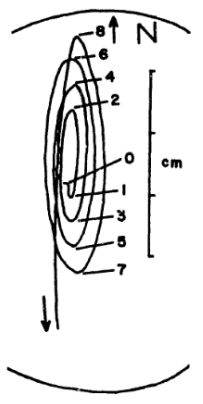

b) Espectros en suelo profundo.

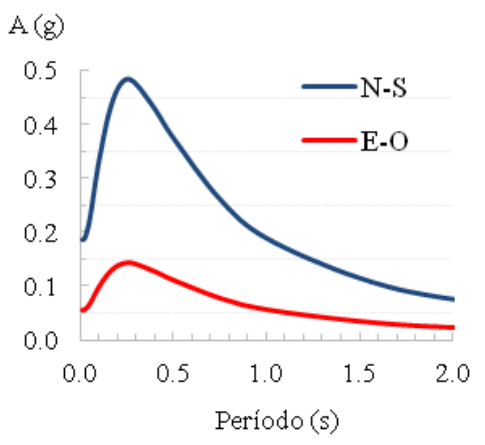

c) Espectros en roca.

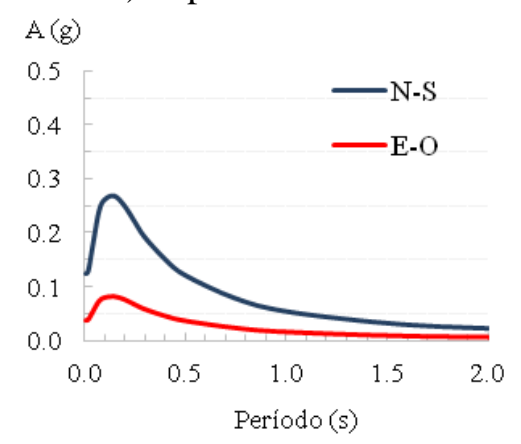

Figura 1. Espectros de aceleraciones (A) en direcciones N-S y E-O, en suelo profundo y en roca, Guerrero y López (2019). El registro del sismoscopio corresponde al adaptado por Robson et al. (1968)

De la base de datos de PEER (2017) se seleccionaron cuatro pares de acelerogramas registrados bajo condiciones similares, tanto tectónicas como locales del sitio de sedimentos profundos en LPG-A. El espectro de cada acelerograma se ajustó al espectro generado en (Guerrero y López, 2019) (Figura 1, Suelo profundo), minimizando la diferencia entre ambos en la banda entre 0,1 y $5 \mathrm{~s}$. Los eventos seleccionados son los NGA 930, 2700, 3908 y 3969, denominados aquí como eventos 1 a 4, respectivamente. La Figura 2 muestra los acelerogramas para los 4 eventos en las dos direcciones horizontales N-S y E-O.

En la Figura 3 se muestra el espectro de aceleraciones para cada uno de los 4 eventos y el espectro suavizado de la Figura 1 (Suelo profundo), para las direcciones N-S y E-O. Para cada dirección, se aprecia que en ciertos períodos puede haber una diferencia apreciable entre las aceleraciones espectrales de los cuatro eventos, así como entre un evento específico y el espectro suavizado que les dio origen. Por ejemplo, se aprecia que el Evento 4 puede ocasionar una respuesta hasta tres veces mayor que el espectro suavizado, para el período de $0,1 \mathrm{~s}$ en dirección E-O. Esta elevada dispersión de la respuesta estructural es una característica bien conocida en la ingeniería sísmica, asociada a las incertidumbres presentes y no controladas en el proceso de selección de los acelerogramas. Es decir, el proceso de simulación en donde se controlan los parámetros de magnitud, mecanismo focal, distancia al plano de falla y condiciones locales de 
rigidez y profundidad de sedimentos, no es suficiente para reducir toda la dispersión implícita en el proceso y persisten diferencias significativas entre las aceleraciones espectrales de un evento a otro.

Dirección N-S
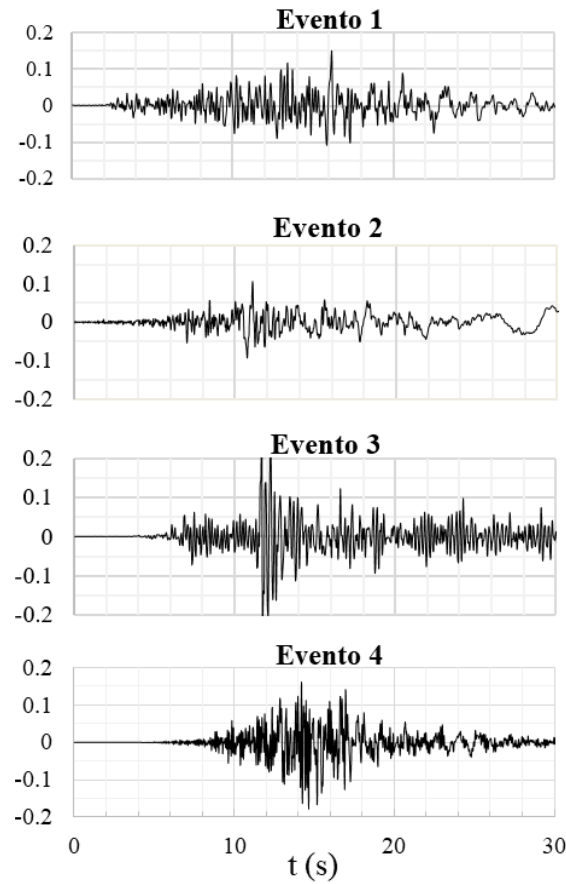

Dirección E-O

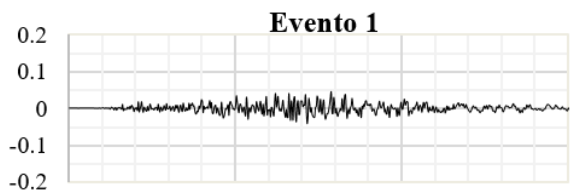

Evento 2

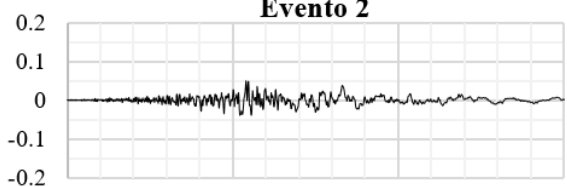

Evento 3

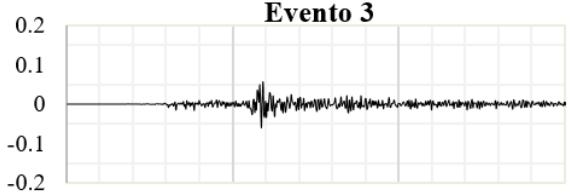

Evento 4

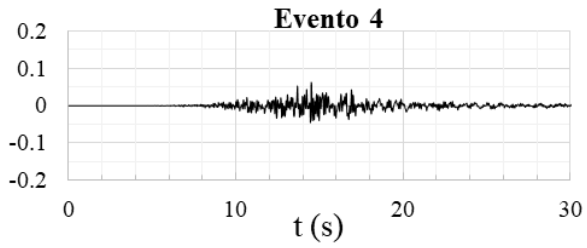

Figura 2. Acelerogramas (g) de los cuatro eventos representativos del sismo de 1967
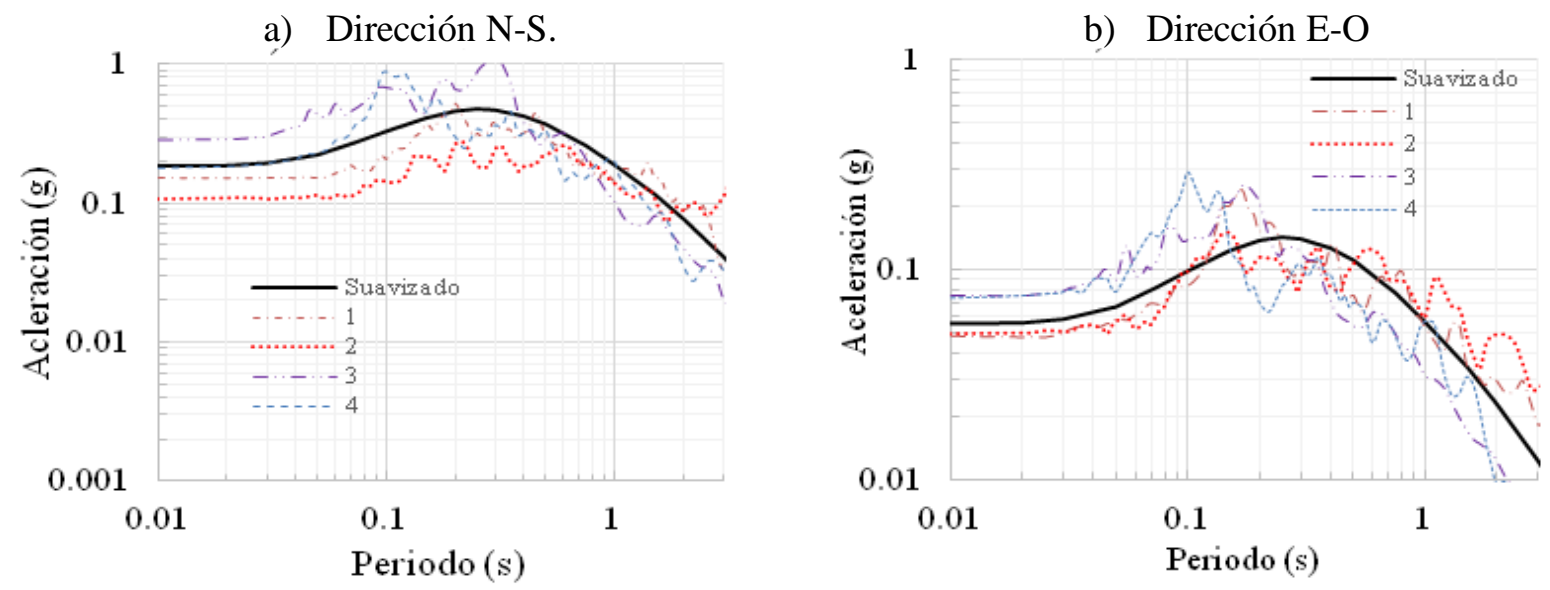

Figura 3. Espectros de Aceleración de los 4 eventos y espectro suavizado de la Figura 1 (suelo profundo) 


\section{DESEMPEÑO DEL EDIFICIO BELAIRE DURANTE EL SISMO DE 1967}

En esta sección se evalúa el desempeño del edificio Belaire ante el movimiento sísmico caracterizado en la sección anterior. El edificio de 9 pisos (Figura 4) está ubicado en la misma zona y tiene las mismas características de vulnerabilidad que los edificios derrumbados, sin embargo, no sufrió ningún daño según CPES (1978). Construido en 1963, tiene alturas de entrepiso de 2,7 m para una altura total $\mathrm{H}=24,3 \mathrm{~m}$, posee una planta cuasi-simétrica y nueve pórticos de concreto armado con vigas altas en la dirección transversal (T) que denominamos dirección Fuerte. En la dirección longitudinal (L) posee cuatro ejes de columnas, sin vigas, la cual denominamos dirección Débil (Figuras 4 y 5). Solo una porción del piso 9 fue construida en concreto y no hay losa del eje 1 al 4 en el último nivel (Figuras 4 y 5). La resistencia del edificio en la dirección L está provista por las columnas que tienen una dimensión constante de $30 \mathrm{~cm}$ en esa dirección, unidas con los nervios de las losas nervadas de $25 \mathrm{~cm}$ de alto separados cada $50 \mathrm{~cm}$. En la dirección fuerte (T) los pórticos poseen vigas altas de sección típica $30 \mathrm{~cm}$ x $50 \mathrm{~cm}$ y secciones de columna de hasta $90 \mathrm{~cm}$ en los niveles inferiores disminuyendo gradualmente hasta tener $30 \mathrm{~cm}$ en el último. Las paredes divisorias internas son de bloques de arcilla de espesor $10 \mathrm{~cm}$ y las paredes externas de $15 \mathrm{~cm}$, las cuales rellenan buena parte de los pórticos con la excepción de la planta baja (PB) lo que genera un entrepiso débil. Mayores detalles se dan en (Guerrero, 2018). El edificio tiene su dirección T (fuerte) orientado N-S que es la dirección de mayor intensidad del sismo de 1967 y su dirección L (débil) en la dirección E-O de menor intensidad.
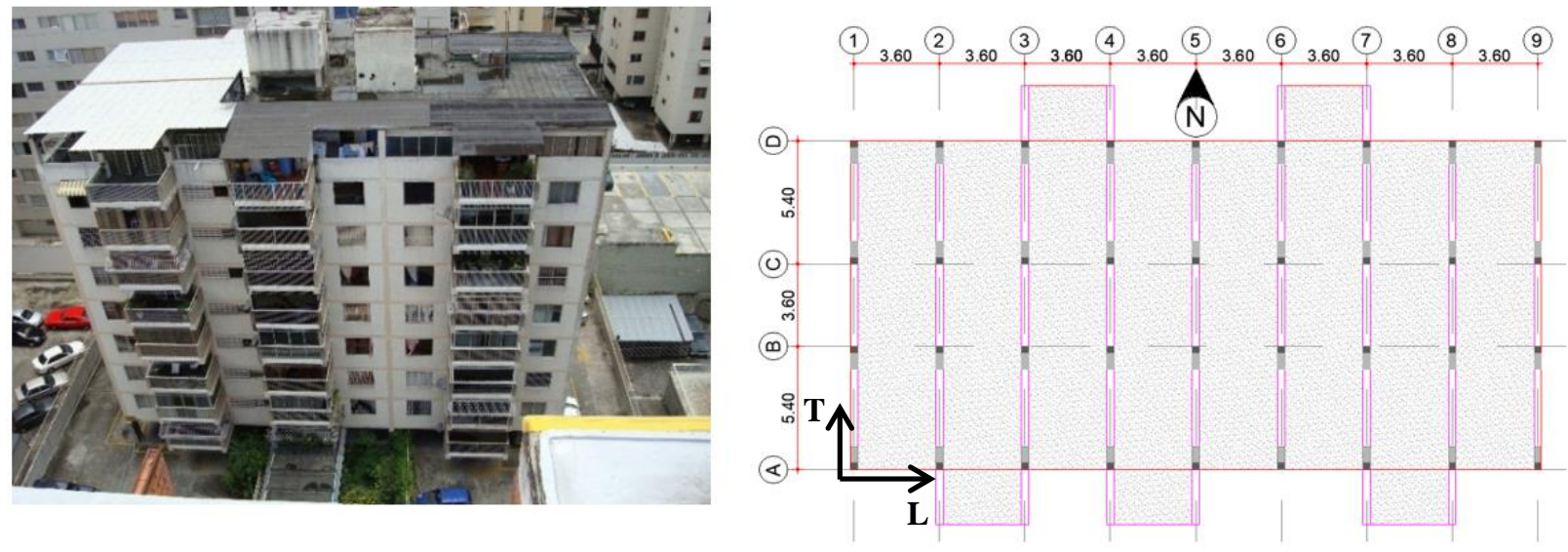

Figura 4. Fachada Sur (izquierda) (Foto cortesía S. Safina) y Esquema de Planta (derecha) del edificio Belaire. La dirección transversal T (fuerte) del edificio corresponde a la dirección N-S

En los planos estructurales no se dan las características de los materiales usados, por lo que, de los ensayos de compresión de 13 cilindros de concreto en distintos elementos, que acompañaban la información disponible en la ingeniería municipal, se obtuvo un máximo y un mínimo de 213 y $178 \mathrm{~kg} / \mathrm{cm}^{2}$, respectivamente. Se estimó una resistencia media de $196 \mathrm{~kg} / \mathrm{cm}^{2}$, valor que fue usado como resistencia a la compresión del concreto. El módulo de elasticidad se estimó en $211.400 \mathrm{~kg} / \mathrm{cm}^{2}$, siguiendo la norma COVENIN 1753 (2006). Al no disponer de información de los aceros del edificio, se usó la información disponible en CPES (1978) en donde se reportan valores de la resistencia cedente de diseño en 21 edificios de LPG-A de $2.800 \mathrm{~kg} / \mathrm{cm}^{2}$, por lo que se estima un esfuerzo cedente promedio un $25 \%$ mayor e igual a $3.500 \mathrm{~kg} / \mathrm{cm}^{2}$, con módulo de elasticidad de $2,1 \times 10^{6} \mathrm{~kg} / \mathrm{cm}^{2}$. Para las paredes de bloques de arcilla, se adoptó una resistencia media a la compresión de $14 \mathrm{~kg} / \mathrm{cm}^{2}$ y un módulo de elasticidad de $29.811 \mathrm{~kg} / \mathrm{cm}^{2}$, basado en ensayos hechos con materiales de esa época (Luchsinger, 1972). 
a) Pórtico 3 en dirección transversal (T).

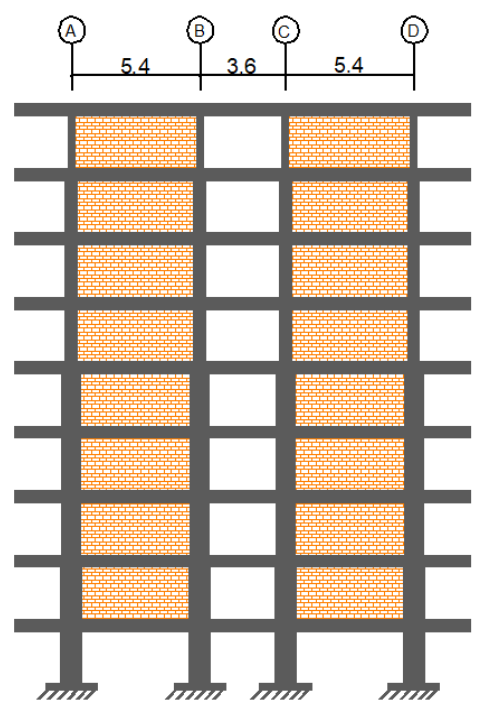

b) Pórtico B en dirección longitudinal (L).

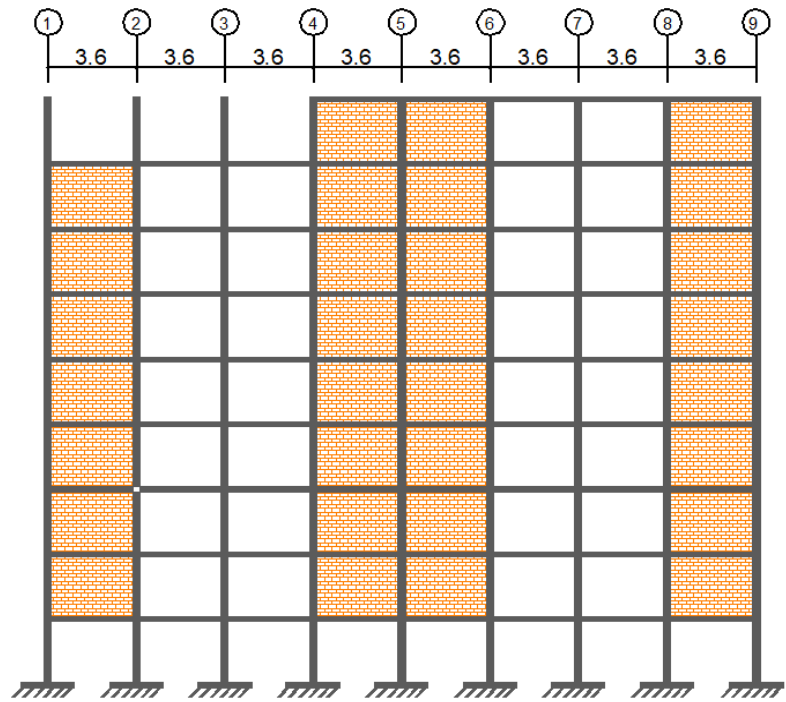

Figura 5. Pórticos representativos y paredes de relleno del edificio, en cada dirección

\section{Medición de propiedades dinámicas en vibración ambiental}

Se realizaron mediciones de vibración ambiental a fin de obtener las propiedades dinámicas básicas del edificio. La vibración del edificio ante acciones ambientales (personas, viento, tráfico, maquinarias) tiene amplitudes pequeñas, la aceleración máxima registrada fue del orden de $10^{-4} \mathrm{~g}$. Se instalaron sensores en los niveles PB, 3, 6, y 8, todos en el centro geométrico de la estructura salvo en el nivel 8 que se agregaron dos equipos en el extremo del edificio para medir la respuesta torsional. En la Figura 6 se muestra una porción de la respuesta medida en la dirección longitudinal en el piso 8, en su centro geométrico, para dos sensores redundantes, los cuales arrojan respuestas coincidentes. La respuesta en el tiempo (Figura 6.a) muestra un período predominante del orden de $0,5 \mathrm{~s}$, el cual se destaca claramente en el pico cercano a $2 \mathrm{~Hz}$ en la densidad espectral de potencia (Figura 6.b) acompañado de un pico de 6,2 Hz de un modo superior. Los resultados indicaron que los periodos principales de vibración son 0,51, 0,38 y 0,33 s, en dirección L, $\mathrm{T}$ y torsional, respectivamente, con un amortiguamiento promedio de $3 \%$.

a) Variación de la aceleración en el tiempo.

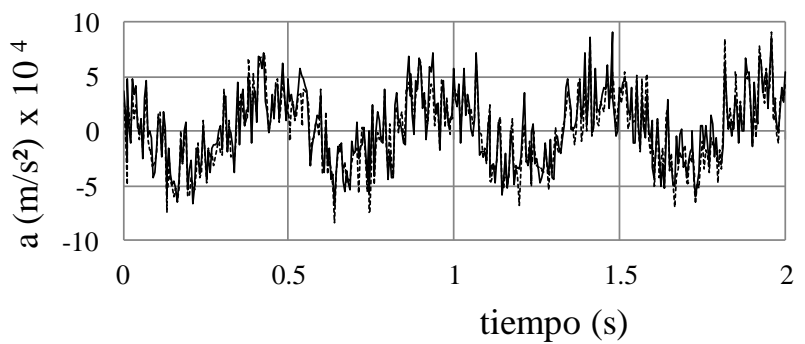

b) Densidad espectral de potencia.

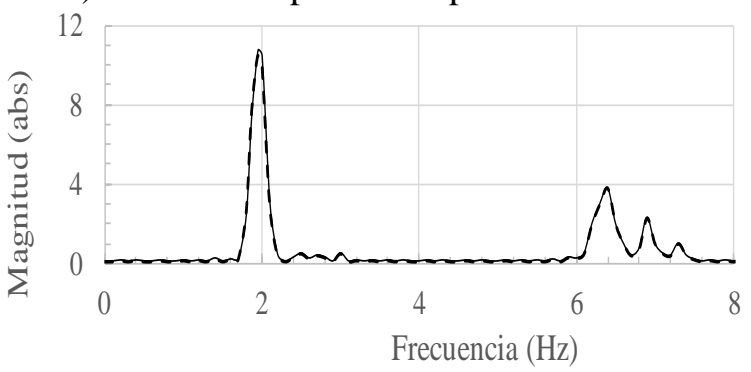

Figura 6. Respuesta dinámica en la dirección longitudinal (L), piso 8, ante excitación ambiental 


\section{Análisis de respuesta espectral}

En una primera etapa se estimó la respuesta máxima del Belaire mediante el método de análisis espectral con la acción sísmica de 1967 descrita por los espectros de la Figura 3, para cada uno de los sismos seleccionados. El edificio se modeló 3D con 3 grados de libertad dinámicos por losa, incorporando la rigidez de la losa fuera de su plano. La losa y la escalera se modelan con elementos de área. Se consideraron todas las cargas permanentes y una carga variable de $50 \mathrm{Kg} / \mathrm{m}^{2}$ para el momento del sismo, con un peso total del edificio de $4.331 \mathrm{t}$. Se desarrollaron 6 modelos matemáticos a fin de evaluar y discriminar el efecto de paredes, escalera, y recubrimientos y acabados, cuyos períodos se muestran en la Tabla 1. El Modelo 1 es el pórtico solo, con sus vigas, columnas y losas. El Modelo 2 incorpora el efecto de la escalera. Los Modelos 3 y 4 incorporan el efecto de las paredes para amplitudes bajas de vibración. El Modelo 4 se denomina Rígido. El Modelo 5 denominado Super-Rígido incluye los efectos del recubrimiento en paredes y acabados de piso en losas y es el de mejor ajuste con los períodos medidos que se muestran en la última fila de la Tabla 1. Comparando de manera progresiva los modelos mostrados de la Tabla 1 se observa el efecto significativo de las paredes, escalera y recubrimientos, los cuales reducen los periodos en relación al pórtico solo (Modelo 1); en el caso particular del Modelo 5, los periodos se reducen 3,7 y 1,8 veces en dirección $\mathrm{L}$ y T, respectivamente. El Modelo 6 representa el efecto de las paredes para amplitudes mayores de vibración, del orden de 1.000 veces las medidas en vibración ambiental, amplitudes esperadas durante la respuesta sísmica; para ello las paredes se modelan como bielas a compresión del espesor de la pared y con un ancho de 0,25 veces la longitud de la diagonal del pórtico; este modelo 6 se le denomina Modelo Flexible.

Tabla 1. Modelos y períodos principales de vibración en cada dirección (segundos)

\begin{tabular}{llccc}
\hline & \multicolumn{1}{c}{ Modelo } & $\mathrm{L}$ & $\mathrm{T}$ & Torsional \\
\hline 1 & Pórticos solo más la losa nervada & 2,12 & 0,75 & 0,78 \\
2 & Modelo 1 más la escalera & 1,77 & 0,74 & 0,78 \\
3 & Modelo 1 más las paredes (elementos de área) & 0,69 & 0,45 & 0,41 \\
4 Rígido & Modelo 1 más escalera y paredes (elementos de área) & 0,67 & 0,45 & 0,41 \\
5 Súper-Rígido & Modelo 4 más recubrimientos y acabados & 0,57 & 0,42 & 0,38 \\
6 Flexible & Modelo 1 más escalera y paredes (bielas a compresión) & 0,81 & 0,53 & 0,50 \\
& Valores medidos en vibración ambiental & 0,51 & 0,38 & 0,33 \\
\hline
\end{tabular}

Se calculó la demanda de deriva en cada entrepiso para cada uno de los 4 sismos, en cada dirección. La deriva de un entrepiso es la diferencia de desplazamientos entre su nivel superior y el inferior, dividida entre la altura de $2,7 \mathrm{~m}$ que los separa y multiplicada por mil. Se obtuvo un valor medio de $0,99 \%$ para el modelo Rígido y de 1,2 \% para el Flexible, aun cuando se obtuvo un valor máximo de 2,2 \%o para un evento, lo que muestra la variabilidad de la respuesta entre eventos similares. Este último valor excede el valor indicado en FEMA (2020) de 1,5 \%o para el inicio de daño leve en estas edificaciones. Se determinó el cociente Demanda/Capacidad a flexo-compresión de todas las columnas de la PB, el promedio para todas las columnas y todos los sismos es de 0,93 para el Modelo Rígido y de 0,72 para el Flexible, indicando que en término medio la demanda está ligeramente por debajo de la capacidad, aun cuando para ciertos eventos se excede la capacidad de algunas columnas. Estos resultados en derivas y cocientes demanda/capacidad no se ajustan estrictamente a la ausencia de daños observada después el sismo y debido a sus limitaciones, motiva al uso de métodos de análisis más refinados para evaluar el desempeño, que se presentan en las siguientes secciones. 


\section{Curvas de capacidad del edificio, análisis estático no lineal (AENL)}

La capacidad del edificio a carga lateral se determinó mediante análisis estático no lineal (AENL) de empuje progresivo (pushover) aplicando una distribución de fuerzas proporcionales al primer modo. Se analizaron dos pórticos representativos del Modelo Flexible (Tabla 1): el pórtico 3 en dirección T y el B en dirección L (Figuras 4 y 5), incorporando en esta dirección una viga equivalente que representa tres nervios de la losa. Los períodos del primer modo de los pórticos son similares a los períodos del edificio. Se definieron rótulas plásticas de baja ductilidad en vigas y columnas, y las paredes se modelan como bielas de carácter frágil, seleccionando las propiedades plásticas de todos los elementos de acuerdo al ASCE 41 (2017). La curva de capacidad que relaciona el cortante basal con el desplazamiento en el último nivel se muestra en la Figura 7, notándose que en la dirección T se produce la primera perdida de rigidez para un desplazamiento de $2,6 \mathrm{~cm}$, asociado a una deriva $(\Delta)$ de $1,1 \%$ (la deriva es el desplazamiento entre la altura del edificio (24,3 m) y multiplicada por mil). Para un desplazamiento (D) de $4,1 \mathrm{~cm}(\Delta=1,7 \%$ ) se produce la primera caída de resistencia debida a la falla de las paredes (entrepisos 2 a 4), posteriormente para $\mathrm{D}=15,6$ $\mathrm{cm}(\Delta=6 \%)$ se genera una falla frágil por corte en las columnas de la PB y la estructura está próxima al colapso. En dirección $\mathrm{L}$, el edificio tiene la primera perdida de rigidez para un $\mathrm{D}=1,55 \mathrm{~cm}(\Delta=0,6 \%$ y $)$ la primera caída de resistencia a $2,5 \mathrm{~cm}(\Delta=1,0 \%$ ), asociadas a los nervios de la losa de la PB. Luego, en $\mathrm{D}=12 \mathrm{~cm}(\Delta=5 \%)$ se han generado daños en paredes y en varios elementos estructurales, las columnas de la PB han alcanzado su resistencia última y el pórtico ha perdido $58 \%$ de su resistencia máxima. En la Figura 7 también se muestra la curva de capacidad de cada pórtico sin incorporar en el modelo las paredes de relleno; nótese que en ambas direcciones las paredes proporcionan mayor rigidez y resistencia al pórtico, siendo esta última hasta 4,5 veces mayor en dirección L. Las paredes también conducen a pérdidas bruscas de resistencia y a una respuesta menos dúctil en ambas direcciones. En dicha figura, se representa en color naranja la respuesta dinámica no lineal del edificio que será descrita posteriormente y la respuesta estática en sentido negativo se adopta igual a la de sentido positivo.

a) Dirección Transversal $\mathrm{T}(\mathrm{N}-\mathrm{S})$

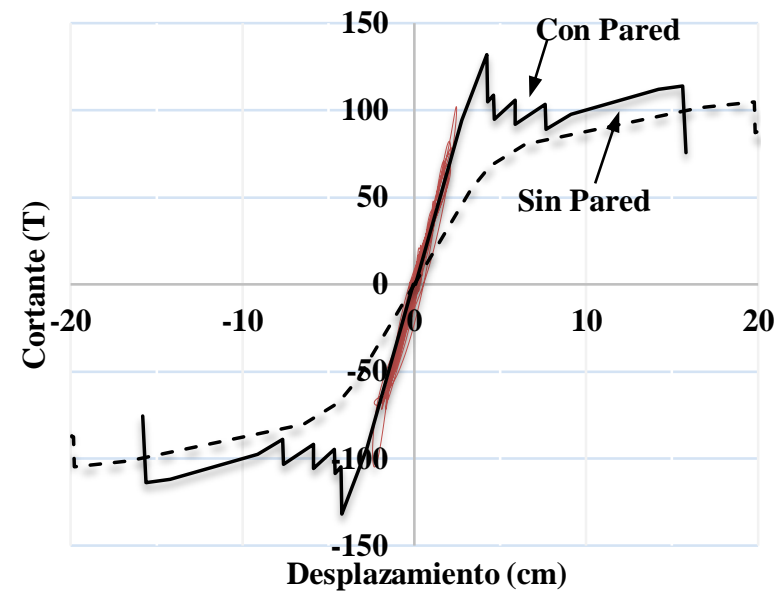

b) Dirección Longitudinal L (E-O)

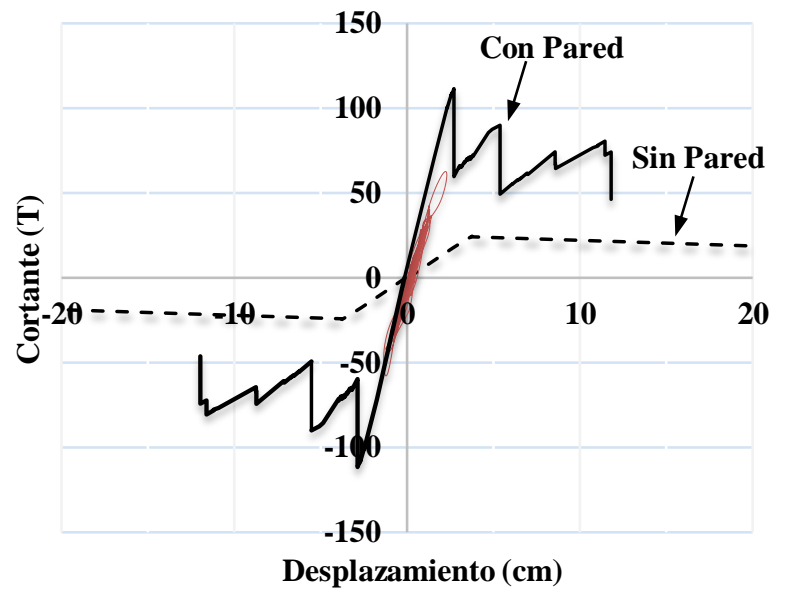

Figura 7. Curva de capacidad (AENL) de pórticos representativos del edificio (negro). Respuesta dinámica (ADNL) (naranja) para el Evento 2, y curva de capacidad del pórtico sin paredes

Las curvas de capacidad mostradas en la Figura 7 describen la respuesta global del pórtico, mostrando la relación entre el cortante en la base y el desplazamiento del último piso. Una descripción más completa que permite discriminar y visualizar el desempeño local, se puede obtener mediante un análisis de empuje 
progresivo (pushover) en cada uno de los entrepisos del pórtico. Las Figuras 8 y 9 muestran la relación entre el cortante y la deriva en los entrepisos 1, 2 y 5 para las direcciones $\mathrm{T}$ y $\mathrm{L}$ del edificio. Las curvas de capacidad de cada entrepiso, se obtuvieron aplicando una carga lateral en un nivel e impidiendo el desplazamiento del nivel inferior, con lo cual se genera un estado de deformaciones concentradas en dicho entrepiso.

El entrepiso 1 (nivel inferior) en ambas direcciones muestra un comportamiento diferente debido a la ausencia de paredes. En los entrepisos superiores, en la dirección L se observa el efecto significativo que tienen las paredes las cuales al fallar provocan una pérdida brusca en la capacidad de carga; en la dirección $\mathrm{T}$ las paredes tienen menor influencia debido a la mayor capacidad de las columnas y a la presencia de vigas.

Se establecieron dos estados de daño a fin de caracterizar el desempeño del entrepiso: El Daño A definido por la articulación de algunas vigas o nervios y a la pérdida de resistencia en paredes y el Daño B definido cuando se alcanza la capacidad última en al menos el 50\% de las columnas del entrepiso, el cual podemos asociarlo a colapso parcial o total. En las Figuras 8 y 9 se muestran las derivas correspondientes a cada estado de daño. En la dirección T donde hay vigas altas (Figura 8), el valor promedio de la deriva para todos los entrepisos en la condición de Daño A es igual a 2,0 \%; como comparación, este valor coincide con el dado por Gobarah (2004) para definir el inicio de daño ligero reparable para pórticos de concreto armado rellenos con paredes, de baja ductilidad; en FEMA (2020) se indican valores entre 1,2 \%о y 2,0 \%o para el inicio de daño ligero en pórticos de concreto con mampostería de relleno no reforzada comprendidos en las categorías Pre-code y Low-code con alturas entre 5 y 12 pisos.
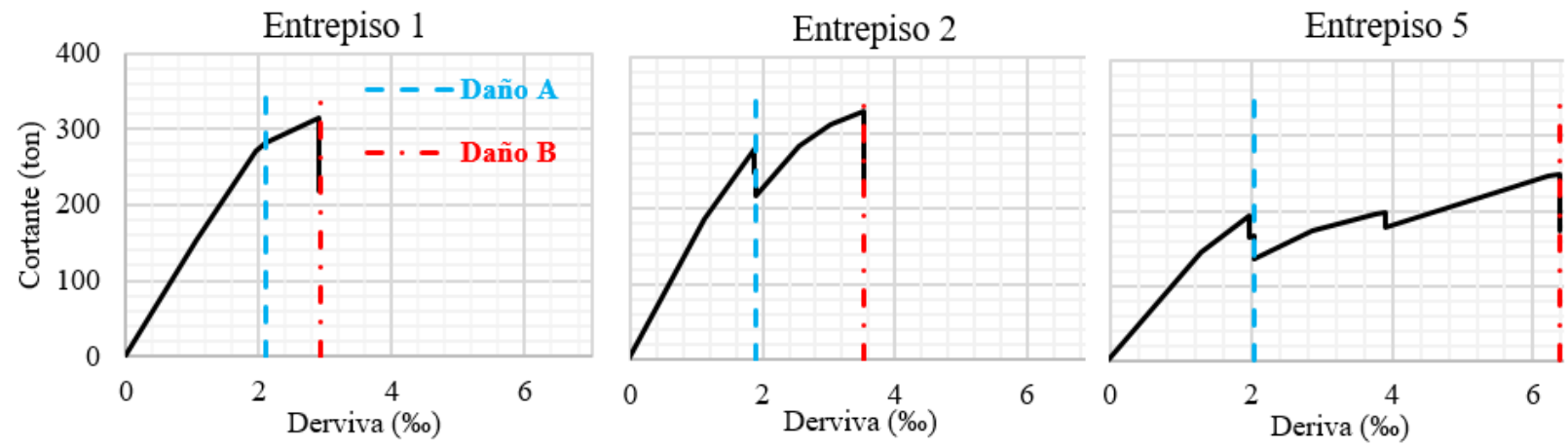

Figura 8. Curvas de capacidad en los entrepisos 1, 2 y 5 y derivas asociadas al Daño A y Daño B en la dirección transversal ( $\mathrm{T}$ ) del edifico (Pórtico 3)
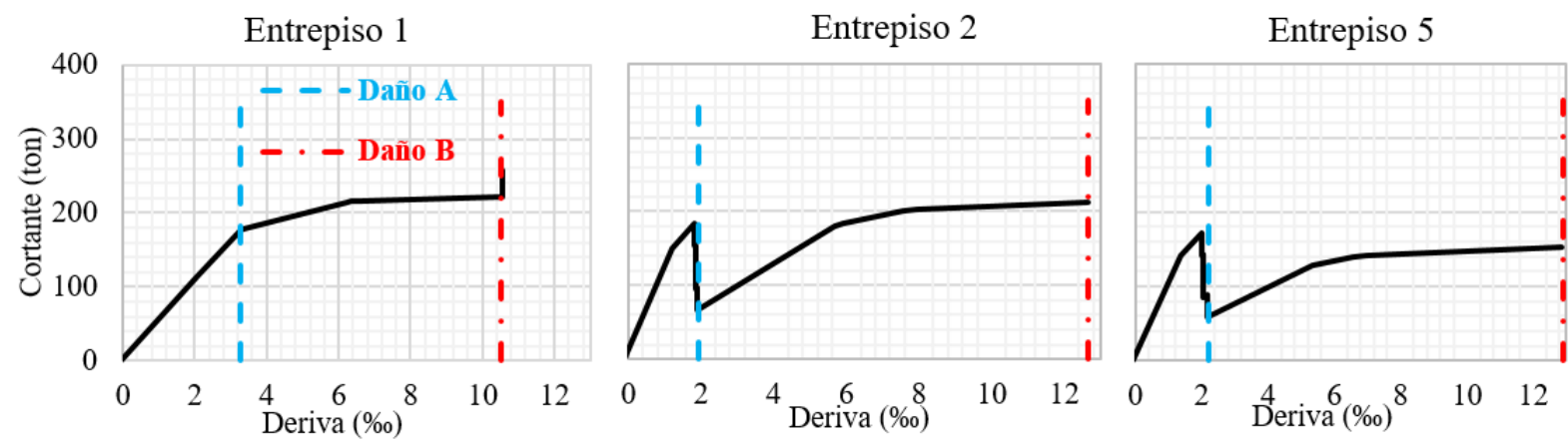

Figura 9. Curvas de capacidad en los entrepisos 1, 2 y 5 y derivas asociadas al Daño A y Daño B en la dirección longitudinal (L) del edificio (Pórtico B) 


\section{Análisis dinámico no lineal (ADNL)}

El desempeño fue evaluado mediante un análisis dinámico no lineal (ADNL) de la estructura sujeta a los cuatro eventos (acelerogramas) definidos previamente (Figura 2). Los acelerogramas N-S se aplican en la dirección fuerte T (Pórtico 3) y los E-O en la dirección débil L (Pórtico B). El modelo de estructura y paredes es el mismo descrito previamente en el AENL con la diferencia de que en este caso se consideró un modelo dúctil que ignora las caídas de resistencia. La intención de este análisis es la determinación de la demanda que impone el movimiento sísmico en términos de desplazamientos y derivas y su comparación con la capacidad estructural determinada previamente. Los cálculos fueron hechos con el programa SAP 2000 .

En la Figura 10 se muestra la historia del desplazamiento en el último nivel en la dirección T, para los eventos sísmicos 2 y 3 que son los que generan menor y mayor desplazamiento, respectivamente, alcanzado un máximo de aproximadamente $3 \mathrm{~cm}$ (Evento 3). La respuesta en dirección L se muestra en la Figura 11 para los eventos 2 y 4, observándose un desplazamiento máximo de 2,3 cm (Evento 2). La relación entre el cortante basal y el desplazamiento en el último nivel, obtenida con el ADNL, se muestra en la Figura 7 en color naranja para el Evento 2, nótese la relación no lineal entre el cortante basal y el desplazamiento, aun cuando el sistema se mantiene dentro del rango elástico lineal, lo cual es debido a la contribución de los modos superiores de vibración; si la respuesta estuviese contenida solo en el primer modo, la relación sería lineal. Sobre dicha curva de respuesta del ADNL se ha superpuesto la curva de capacidad del AENL, presentada previamente. Se observa que la fuerza cortante y las deformaciones generadas durante la respuesta dinámica para el Evento 2 están por debajo de los valores que inducirían daño, para cada dirección.
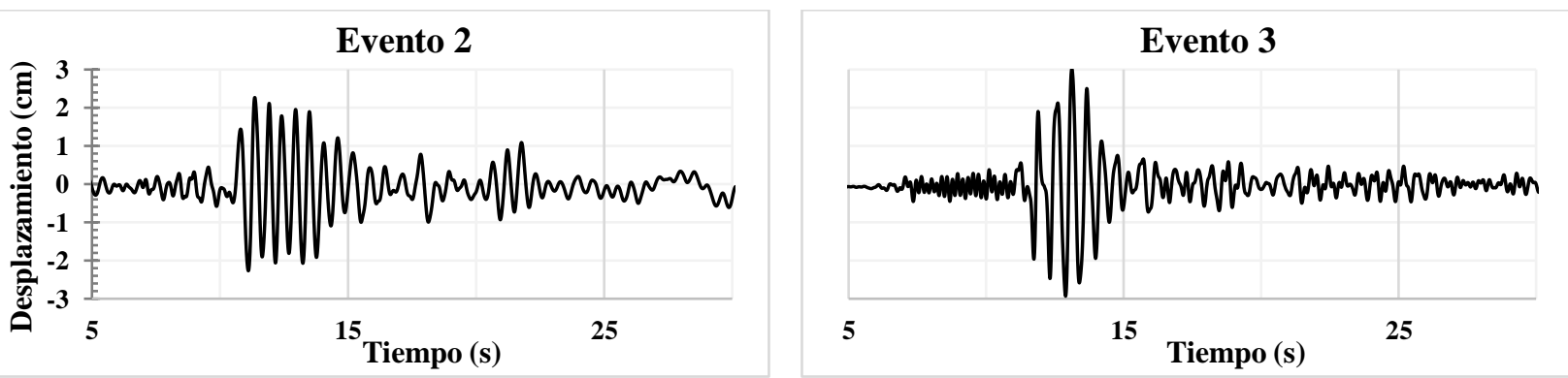

Figura 10. Historia del desplazamiento en el último nivel, dirección transversal (T) del edificio para los eventos sísmicos 2 y 3
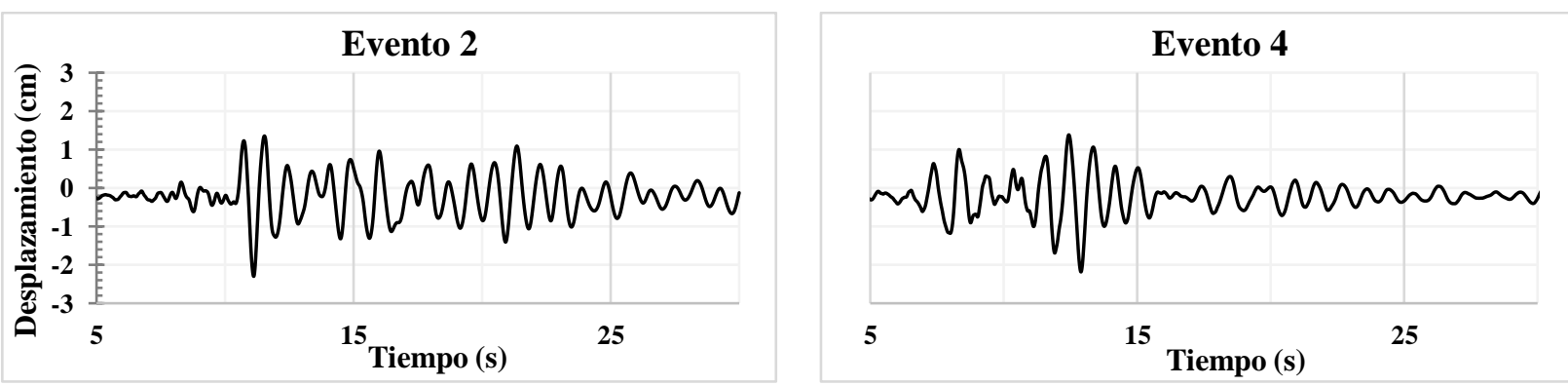

Figura 11. Historia del desplazamiento en el último nivel, dirección longitudinal (L) del edificio para los eventos sísmicos 2 y 4 
La Figura 12 muestra las derivas máximas en cada entrepiso obtenidas del ADNL, en las mismas figuras se indica la deriva asociada a los estados de Daño A y B. La deriva en todos los entrepisos para los eventos 1, 2 y 4 es menor a la asociada con Daño A. La mayor demanda en la dirección T se produce en los entrepisos inferiores cuando la estructura está sometida al evento 3 y $\Delta$ vale $2,1 \%$, valor coincidente con la ocurrencia del Daño A. En la dirección L todas las derivas son inferiores a la del Daño A, lo cual concuerda con lo observado en 1967. Para la dirección L el mayor valor de $\Delta$ es de 1,5\% en la planta baja y corresponde a los eventos 1 y 2 ; el evento 3 produce una demanda de $0,8 \%$.

Los resultados discutidos previamente se corresponden con lo observado; el edificio Belaire no ha debido sufrir daño estructural ni daño significativo en paredes durante el sismo de 1967. Las aceleraciones máximas de los 4 acelerogramas en dirección Este-Oeste (Figura 2) están comprendidas entre 0,048 g y $0,075 \mathrm{~g}$, que son aproximadamente 2 a 3 veces mayores que las predichas en el análisis simplificado previo del edificio (Urich y López, 2006).

Por otro lado, se pone de manifiesto la diferencia entre las respuestas dadas por distintos acelerogramas, aun cuando todos ellos fueron generados bajo las mismas condiciones tectónicas y locales de sitio.
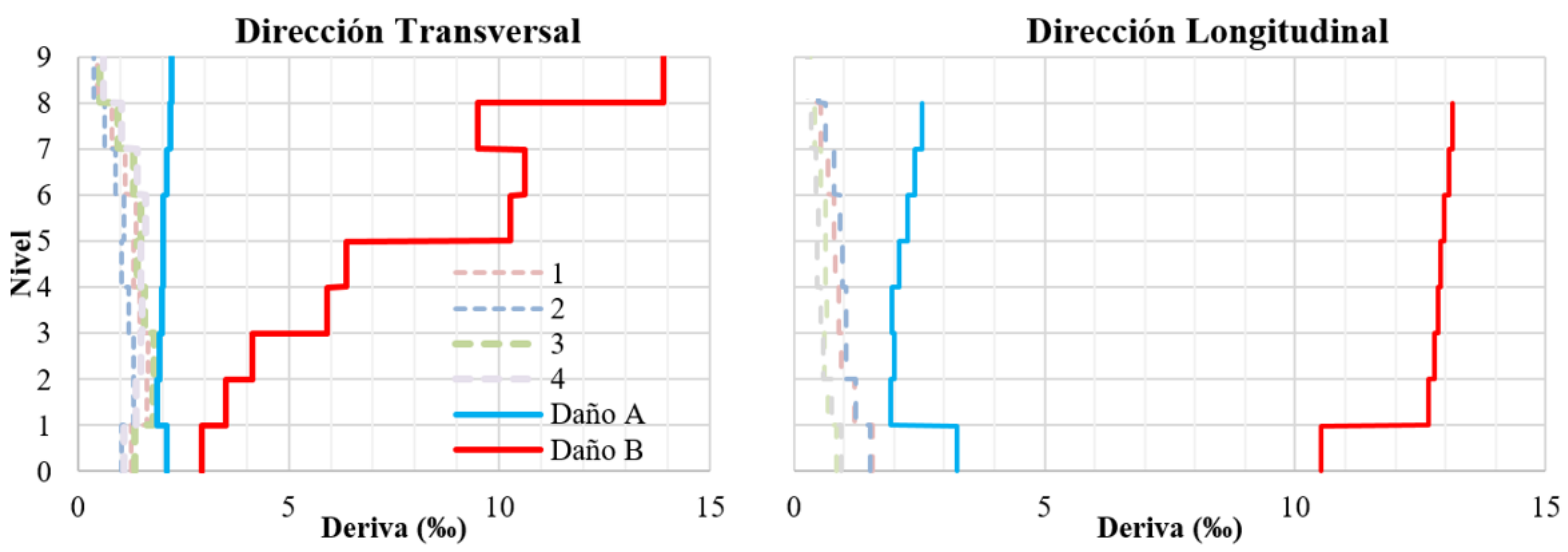

Figura 12. Comparación de la demanda de deriva en el edificio por evento (1 a 4) con las derivas asociadas al Daño A y Daño B, para cada entrepiso

\section{Respuesta del edificio Belaire rotado 90 grados}

Se presenta la respuesta del edificio rotado 90 grados, es decir, la dirección débil (L) se hace coincidir ahora con la dirección N-S de mayor intensidad sísmica (Figura 1). Se muestra en la Figura 13 la respuesta en el tiempo asociada al desplazamiento en el último nivel y la deriva en el nivel planta baja, en dirección longitudinal (L), para el edificio en condición original y para el edificio rotado. Al compararlos se observa cómo se genera una demanda de desplazamiento y de deriva hasta 3 veces mayor en el edificio rotado. También se aprecia una deriva máxima de 4\%o que supera el límite de Daño A y una deformación remanente que revela incursiones en el rango inelástico en el edificio rotado. 
a) Desplazamiento en el último nivel

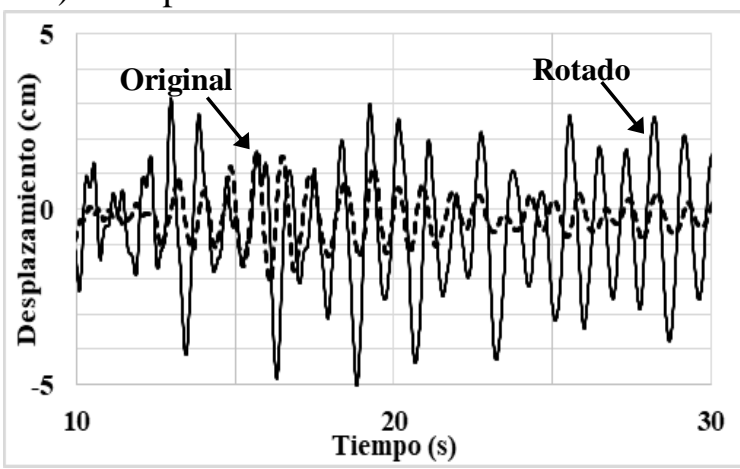

b) Deriva (\%o) en el nivel inferior (PB)

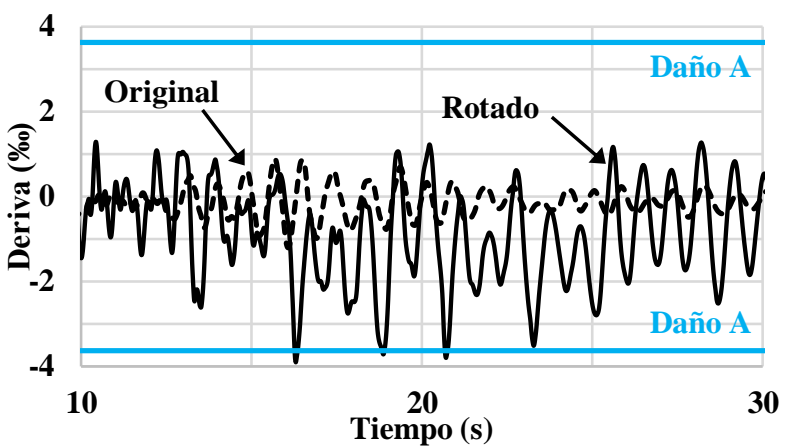

Figura 13. Historia de desplazamiento y deriva en dirección longitudinal (L) para el edificio en condición original y rotado $90^{\circ}$, ante el evento 1

La Figura 14 muestra la demanda de deriva en cada entrepiso para cada dirección y evento sísmico, obtenidas del ADNL; se muestran también las derivas asociadas a los estados de Daño A y B en cada entrepiso. Al comparar la Figura 14 con la 12 se aprecia el efecto de rotar el edificio. En la dirección L del edificio rotado se excede ahora el nivel de Daño A en los dos entrepisos inferiores, para todos los eventos. La deriva en dirección L alcanza el $4 \%$ y al menos para un evento se excede la resistencia de las paredes, se produce la rotura de la losa en los entrepisos 2 a 6 y se alcanza la resistencia cedente de las columnas de la planta baja. El daño se puede catalogar como daño estructural moderado.
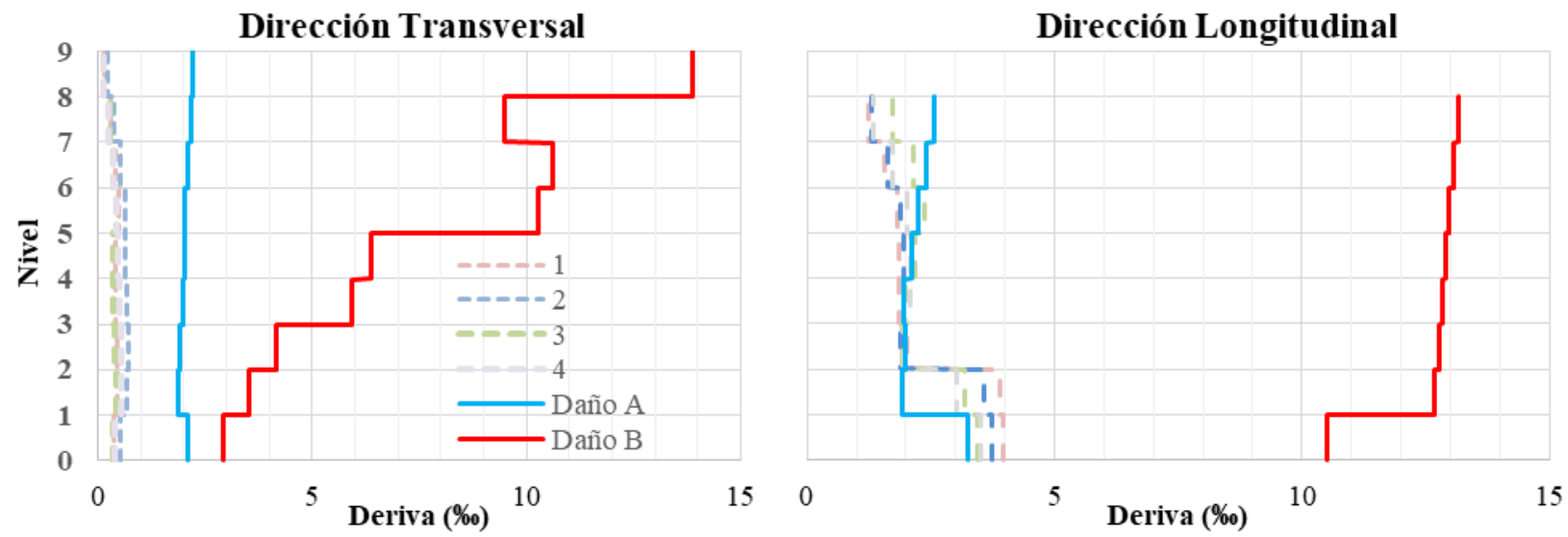

Figura 14. Edificio rotado $90^{\circ}$. Comparación de la demanda de deriva por evento (1 a 4 ) para el sismo de 1967 con las derivas asociadas al Daño A y Daño B, para cada entrepiso

\section{CONCLUSIONES}

Se realizaron mediciones de vibración ambiental del edificio Belaire obteniéndose información de las propiedades de la edificación lo que sirvió para calibrar los modelos matemáticos. Del análisis de respuesta espectral se observa, a partir de la disminución de los periodos, la influencia significativa de las paredes y la escalera en la rigidez de la estructura. Mediante el análisis estático y dinámico no lineal se estudió en detalle el edificio, el cual no sufrió ningún daño durante el sismo de 1967 aun cuando poseía una estructura y antigüedad similar a los edificios dañados en esa zona. El análisis se efectúa con los acelerogramas de 
cuatro eventos representativos del movimiento sísmico; el movimiento en la dirección de mayor intensidad $(\mathrm{N}-\mathrm{S})$ se aplicó en la dirección fuerte (T) del edificio y el movimiento en la dirección de menor intensidad (E-O) en la dirección débil (L). La condición de piso débil que existe en PB por la discontinuidad de paredes no se manifestó debido a que la estructura respondió en el rango elástico dada la coincidencia favorable de la dirección fuerte $(\mathrm{T})$ del edificio y la dirección de mayor intensidad sísmica. Del análisis se observa que ninguno de los eventos genera daño estructural, lo cual coincide con lo observado y ratifica la representatividad de los movimientos sísmicos utilizados.

Se determinó el comportamiento que hubiese tenido si el edificio hubiese estado rotado $90^{\circ}$, haciendo coincidir su dirección más débil (L) con la dirección de mayor intensidad (N-S) del sismo, la cual es aproximadamente tres veces más intensa. Para todos los eventos, se obtiene que la estructura tendría daños significativos: se excede la capacidad cedente de las columnas del nivel inferior y ocurren fallas en nervios de las losas y agrietamiento importante en paredes. Se observa una concentración de la demanda (dirección L) en los dos primeros entrepisos debido a la discontinuidad de las paredes en PB que generan un piso débil. Se corrobora que el edificio Belaire no sufrió daños en 1967 por tener su dirección más fuerte orientada en la dirección Norte-Sur, que coincidió favorablemente con la dirección de mayor intensidad del sismo. Tomando en cuenta que en sismos futuros se pueden esperar intensidades aproximadamente similares en cualquier dirección, se concluye la necesidad de reforzar edificios antiguos similares en Caracas aun cuando ellos no sufrieron daños en 1967 por esta condición particular de orientación.

Se destaca que la respuesta del edificio varía significativamente de un evento sísmico a otro, a pesar de que ellos fueron generados con los mismos parámetros sismológicos y geotécnicos. Pequeñas diferencias en los acelerogramas (y sus espectros), influencian significativamente la respuesta. En 1967 unos edificios tuvieron daño estructural, pero otros no, a pesar de ser prácticamente iguales, lo cual se puede explicar reconociendo la influencia que tiene una pequeña variación del período de vibración en la sincronización favorable o desfavorable que puede ocurrir entre la respuesta y el movimiento sísmico, lo cual, en ciertos casos pudiera significar la diferencia entre la supervivencia o el colapso de una estructura.

\section{AGRADECIMIENTOS}

Se agradece el apoyo del IMME-FI-UCV y de FUNVISIS a través del Proyecto Sismo Caracas donde se desarrolló parte de esta investigación, así como al Profesor Simón Morales por su apoyo en el procesamiento de vibraciones ambientales y al Ing. Alfredo Urich por facilitarnos los planos del edificio.

\section{REFERENCIAS}

ASCE (2017). Seismic rehabilitation of existing buildings. ASCE 41. American Society of Civil Engineers. Virginia, EEUU. DOI: 10.1061/9780784408841

Bozorgnia, Y, N Abrahamson, L A Atik, T Ancheta, G Atkinson, J Baker, A Baltay, D Boore, K Campbell, B Chiou, R Darragh, S Day, J Donahue, R Graves, N Gregor, T Hanks, I Idriss, R Kamai, T Kishida,

A Kottke, S Mahin, S Rezaeian, B Rowshandel, E Seyhan, S Shahi, T Shantz, W Silva, P Spudich, J Stewart, J Watson-Lamprey, K Wooddell y R Youngs (2014). "NGA-West2 Research Project". Earthquake Spectra, Vol. 30, No. 3, pp. 973-987. DOI: 10.1193/072113EQS209M

COVENIN 1753 (2006). Norma Venezolana: Proyecto y construcción de obras en concreto estructural. FONDONORMA Caracas, Venezuela. 
CPES (1978). Segunda fase del estudio del sismo ocurrido en Caracas el 29 de julio de 1967 (Vol A y Vol. $B$ ). Comisión Presidencial para el Estudio del Sismo, Caracas, FUNVISIS.

FEMA (2020). Hazus earthquake model technical manual. Federal Emergency Management Agency (FEMA). Washington D.C., USA. https://www.fema.gov/sites/default/files/202010/fema_hazus_earthquake_technical_manual_4-2.pdf

Ghobarah, A (2004). "On drift limits associated with different damage levels". International Workshop on Performance-Based Seismic Design. Department of Civil Engineering, McMaster University, Ontario, Canada.

Grases, J (1968). “El sismo de Caracas de 1967. "Estudios orientados hacia su interpretación a partir del comportamiento de estructuras". Boletín Técnico IMME, 6 (23-24), 3-125.

Guerrero A y O A López (2019). "Espectro de respuesta del sismo de Caracas de 1967”. Rev. Téc. Ing. Univ. Zulia. Vol. 42, No. 3, 2019, Septiembre-Diciembre, pp. 104-151. DOI: 10.22209/rt.v42n3a01

Guerrero, A (2018). "Desempeño de edificios afectados por el sismo de Caracas de 1967 en la zona de Los Palos Grandes- Altamira". Trabajo de Grado de M. Sc., Tutor: Oscar A. López.

Luchsinger, J (1972). “Contribución de los elementos de tabiquería de arcilla en la respuesta de estructuras sometidas a cargas sísmicas”. Boletín Técnico del IMME (N 39-400).

PEER. Pacific Earhquake Ground Motion Database. Consultada en octubre de 2017. https://ngawest2.berkeley.edu

Robson, G R, L Canales y L Esteva (1968). "The Caracas earthquake of 29 July 1967: Venezuela”, Serial № 571/BMS.RD/AVS. UNESCO. Paris. https://unesdoc.unesco.org/ark:/48223/pf0000015201_eng

Schmitz, M, J Hernández, C Morales, J Domínguez, V Rocabado, M Vallée, y otros (2011). "Principales resultados y recomendaciones del proyecto de microzonificación sísmica en Caracas y estrategias para su implementación”. Rev. Fac. de Ing. U.C.V., 26 (2), 113-128. Recuperado el 27 de enero de 2021, de http://ve.scielo.org/scielo.php?script=sci_arttext\&pid=S0798$\underline{40652011000200011 \& \operatorname{lng}=\mathrm{es} \& \operatorname{tlng}=\mathrm{en}}$

Skinner, R I (1969). "Damage mechanisms and design lessons from Caracas". Proceedings of the Fourth WCEE, Vol. 3, J-2. Santiago de Chile. 124 a 136.

Sozen, M A, P C Jennings, R Matthiensen, y G W Housner (1968). "Engineering report on the Caracas earthquake of July 29, 1967”. Washington National Academy of Sciences. 116.

Urich, A y O A López (2006). "Importancia de la orientación de los planos resistentes en el desempeño sísmico de edificios en Los Palos Grandes 1967”. VIII CONVESIS, Valencia.

Urich, A (2013). “Desempeño de edificios y acciones sísmicas en los Palos Grandes 1967”. Tesis de M.Sc. Tutor: Oscar A. López. Universidad Central de Venezuela. Fac. de Ingeniería. 